\title{
O EFEITO DA PRÁTICA REGULAR DE EXERCíCIOS FÍSICOS NO ESTILO DE VIDA E DESEMPENHO MOTOR DE CRIANÇAS E ADOLESCENTES ASMÁTICOS
}

Andressa Ribeiro Contreira

Universidade Federal de Santa Maria, Santa Maria, Rio Grande do Sul, Brasil

Sarita Nascimento de Salles

Universidade Federal de Santa Maria, Santa Maria, Rio Grande do Sul, Brasil

Mirela Pereira da Silva

Universidade Federal de Santa Maria, Santa Maria, Rio Grande do Sul, Brasil

\section{Danielle Ledur Antes}

Universidade Federal de Santa Catarina, Florianópolis, Santa Catarina, Brasil

\section{Juliana Izabel Katzer}

Universidade Federal de Pelotas, Pelotas, Rio Grande do Sul, Brasil

\section{Sara Teresinha Corazza}

Universidade Federal de Santa Maria, Santa Maria, Rio Grande do Sul, Brasil

\section{Resumo}

Este estudo objetivou verificar os efeitos de um programa de 10 semanas de ginástica respiratória e natação no estilo de vida, desempenho motor e no comportamento do fluxo expiratório de crianças e adolescentes asmáticos. Participaram do estudo 13 indivíduos com idade média de 11,09 $\pm 2,24$ anos, de ambos os sexos. Para avaliação do estilo de vida, utilizou-se um questionário com questões referentes ao uso de medicações, disposição para prática de atividades físicas, melhora do sono, entre outras. Para verificação do comportamento do fluxo expiratório, utilizou-se um expirômetro e para análise do desempenho motor a Bateria de Testes de Bruininski e Ozerestsky. Os resultados revelaram melhoras nos elementos do estilo de vida, conforme a percepção dos responsáveis, e discreta melhora do fluxo expiratório após o programa. Quanto ao desempenho motor, não houve diferença estatisticamente significativa após o programa.

Palavras-chave: Asma - Crianças - Atividade Física - Estilo de Vida - Desempenho Motor

\section{Introdução}

Nos últimos anos, houve um aumento significativo das doenças 
por problemas respiratórios afetando as vias aéreas, dentre estas doenças encontra-se a asma como a de maior incidência. De acordo com a Sociedade Brasileira de Pneumologia e Tisiologia (2002), a prevalência média da asma no Brasil é de $20 \%$, sendo a quarta maior causadora de hospitalização no País.

A asma é definida como uma doença crônica e de caráter recorrente que acomete as vias aéreas tornando-as hiperirritáveis e hipersensíveis, atingindo indivíduos de todas as idades e de ambos os sexos, implicando em modificações de algumas dimensões da vida do indivíduo, tais como o sono, os hábitos alimentares, o aspecto emocional, bem como agravos à saúde (CABRAL; TEIXEIRA, 1994; GUALDI; TUMELERO, 2004; PITANGA, 2004; TAKETOMI; MARRA; SILVA, 2005; REZENDE et al., 2006; BROCKMANN et al., 2007).

Alguns estudos (GUALDI; TUMELERO, 2004; SILVA et al., 2005; MOISÉS, 2006; REZENDE et al., 2006; MORAES et al., 2007; BROCKMANN et al., 2007) observaram que pacientes com doença respiratória também tendem a mostrar menor tolerância ao exercício físico devido à dificuldade para respirar, restrição à prática de atividades ou mesmo à falta de atividades físico-motoras. Esta privação deve-se à existência de mitos acerca da asma em relação à prática de atividade física, havendo uma espécie de ausência de experiências motoras a fim de evitar a ocorrência de crises de asma. Algumas atividades cotidianas, como correr, andar de bicicleta ou fazer Educação Física na escola, são praticamente excluídas do repertório motor das crianças que possuem asma. Esta falta de oportunidades para a prática tende a prejudicar a aquisição de um estágio maduro das habilidades, prejudicando o processo de desenvolvimento motor, que é fundamental para o refinamento das capacidades motoras e aquisição de hábitos saudáveis que podem ser incorporados por toda a vida (MOISÉS, 1993; CABRAL; TEIXEIRA, 1994; GALLAHUE; OZMUN, 2005; BROCKMANN et al., 2007).

De acordo com Betio, Krebs e Keulen (2007), é indispensável a conscientização dos pais e crianças sobre a importância da atividade física para o desenvolvimento dos aspectos físicos, cognitivos, emocionais, sociais e motores, auxiliando no tratamento da asma. Dessa forma, cabe aos profissionais de Educação Física ficar atentos aos sintomas apresentados pelos alunos durante as aulas, bem como avaliar essas crianças a fim de elaborar programas motores que potencializem seu desenvolvimento. 
Pesquisas revelam que programas de atividade física voltados para essa patologia tendem a reduzir o número de ocorrência de crises de asma e promovem uma boa ventilação pulmonar, podendo ser indicados desde que combinados ao uso de medicamentos (SILVA; SANTOS; MIRANDA, 1994; CASSOL et al., 2004; SILVA et al., 2005; MOISÉS, 2006; REZENDE et al., 2006; MORAES et al., 2007; BETIO; KREBS; KEULEN, 2007; ANTES et al., 2008). Ainda, as atividades físicas são provedoras de ganhos na mecânica respiratória, na prevenção e correções posturais, melhorando a condição física geral dos indivíduos asmáticos e contribuindo para o desenvolvimento das aptidões físicas e motoras (CABRAL; TEIXEIRA, 1994).

Nesse contexto, cabe destacar que os exercícios podem ser classificados como "mais asmagênicos" (provocadores de crises), entre eles a corrida, a caminhada forte, o andar de bicicleta, e também os "menos asmagênicos", como a natação. Esta atividade é, portanto, a mais recomendada aos asmáticos por proporcionar um ambiente úmido e adequado ao bom funcionamento da função respiratória e ventilação pulmonar, reeducação diafragmática, fortalecimento da musculatura respiratória e corporal geral e prevenção de alterações na coluna vertebral (MOISÉS, 1993; WEISGERBER et al., 2003). Dessa forma, a prática da natação em suas diversas modalidades é preconizada para crianças asmáticas, pois exige adaptações na maneira de respirar, estimulando a correção das deformações que atingem a caixa torácica.

Com base nessas informações, e na ausência de estudos que contemplem em conjunto a avaliação das variáveis motoras e do estilo de vida, justifica-se este estudo que busca verificar os efeitos de um programa de ginástica respiratória e natação no estilo de vida e desempenho motor de crianças e adolescentes asmáticos.

\section{Métodos}

Esta pesquisa foi realizada de acordo com a resolução $n^{\circ}$ 196/96 do Conselho Nacional de Saúde (CNS) e aprovada em seus aspectos éticos e metodológicos pelo Comitê de Ética e Pesquisa da Universidade Federal de Santa Maria (CEP/UFSM) sob protocolo $\mathrm{n}^{\circ}$ 0193.0.243.000-07. Antes da realização dos testes, os responsáveis pelas crianças e adolescentes leram e assinaram o Termo de Consentimento Livre e Esclarecido sobre os trâmites da pesquisa. 


\section{Sujeitos}

O grupo de estudo foi composto por 13 indivíduos (crianças e adolescentes), de ambos os sexos (8 meninas; 5 meninos) com diagnóstico de asma, participantes iniciantes do projeto de extensão "Natação e ginástica respiratória para crianças e adolescentes com problemas respiratórios", vinculado ao Centro de Educação Física e Desportos da Universidade Federal de Santa Maria (CEFD/UFSM). A faixa etária fixada foi de 7 a 14 anos (média 11,09 $\pm 2,24$ anos), devido ao protocolo de testes motores (04 - 14 anos). Foram excluídos do estudo crianças e adolescentes com frequência inferior a $75 \%$ das aulas e com faixa etária não condizente com a solicitada no presente estudo. $\mathrm{O}$ grupo de estudo inicial foi composto por 17 crianças, mas, devido às faltas nas aulas, o grupo final foi composto de 13 participantes. O tipo de asma presente no grupo era asma leve caracterizada por crises esporádicas e que melhoram com o uso de broncodilatadores. No período entre as crises, a pessoa é assintomática, tendo boa tolerância ao exercício físico e vida normal (MOISÉS, 1993). O tipo de asma foi comprovado por meio de atestado médico do pneumologista no momento de matrícula no referido projeto.

\section{Instrumentos}

Para a análise dos componentes do estilo de vida, aplicou-se um questionário elaborado para os fins específicos deste estudo, referente à percepção dos pais sobre as modificações no estilo de vida dos participantes após 10 semanas de tratamento. O referido questionário foi entregue aos responsáveis para que acompanhassem as alterações no decorrer do período de intervenção. Os componentes analisados foram: melhora no problema respiratório, sono, respiração, uso de medicação, alimentação, disposição para outras atividades ou para a prática de atividades físicas (incluindo a participação nas aulas de Educação Física na escola).

O instrumento utilizado para verificação do fluxo expiratório foi o expirômetro modelo Mini-Wright-Peak Flow Meter 3103, marca Clement Clarke. As medidas do fluxo expiratório, ao contrário das outras avaliações, foram realizadas uma vez a cada semana, durante 7 semanas. Para a realização da medida, o aluno deveria permanecer descansado e em pé, segurando o aparelho de maneira a não obstruir o 
orifício de saída do ar, devendo inspirar o máximo possível e então colocar a boca no bocal e expirar o mais forte possível. Foram realizadas 3 tentativas e foi considerada a melhor delas, ou seja, a que apresentava maior medida do fluxo expiratório, conforme consta no protocolo de avaliação.

Utilizou-se como instrumento para a análise do desempenho motor dos participantes a Bateria de Testes de Bruininski e Ozerestsky (1978 apud GALLAHUE, 1994). O instrumento informa sobre as aquisições motoras de crianças e jovens, não só avaliando funções como também as disfunções e atrasos de desenvolvimento motor. A forma utilizada neste estudo consta de 14 subtestes que medem os seguintes itens: agilidade e velocidade; equilíbrio (estático e dinâmico); coordenação bilateral; força; coordenação de membros superiores; velocidade de reação ou tempo de reação; controle visomotor e destralidade. Os valores de proficiência motora geral resultaram da soma da pontuação de todos os itens, de acordo com a faixa etária em meses de cada participante, conforme consta no manual da Bateria de Testes anteriormente citada.

O programa de treinamento proposto foi desenvolvido no período de 10 semanas, de 2 aulas semanais com duração de 50 minutos cada sessão, sendo 20 minutos destinados ao desenvolvimento da ginástica respiratória e o tempo restante destinado ao desenvolvimento da natação. O primeiro momento do programa (ginástica respiratória) constou de exercícios visando o relaxamento muscular, exercícios respiratórios, fortalecimento muscular e alongamentos, segundo a rotina de atividades do projeto de extensão. Já a natação compreendia o trabalho de adaptação/familiarização ao meio líquido, iniciação aos 4 estilos (crawl, costas, peito e golfinho) e atividades lúdicas. As aulas foram desenvolvidas nas piscinas térmicas do Centro de Educação Física e Desportos (CEFD/UFSM).

A coleta de dados das avaliações motoras foi realizada no Laboratório de Aprendizagem Motora do CEFD/UFSM e os testes de expirometria e aplicação dos questionários foram realizados antes das aulas e ocorreram no próprio ambiente das piscinas térmicas. Após 10 semanas de aulas de natação, foram realizados os pós-testes da avaliação motora e questionários, enquanto os dados do comportamento do fluxo expiratório foram realizados em 7 semanas de intervenção.

Para análise dos resultados obtidos em pré e pós-teste utilizou-se uma estatística descritiva (média e desvio-padrão) e em percentis, o 
Teste $\mathrm{t}$ foi utilizado para verificação das diferenças entre pré e pós-teste, mediante o Pacote Estatístico SPSS, versão 11.0 com nível de significância de $\mathrm{p}<0,05$.

\section{Resultados e discussão}

O Quadro 1 refere-se às variáveis do questionário dos componentes do estilo de vida, indicando a presença de melhora ou não dessas variáveis. As respostas foram relativas às percepções dos responsáveis acerca das mudanças nos componentes do estilo de vida dos participantes após a participação no programa.

\begin{tabular}{c|c|c}
\hline VARIÁVEL & MELHOROU & NÃO MELHOROU \\
\hline Problem a respiratório & $\mathbf{1 0 0 \%}$ & - \\
\hline Sono & $\mathbf{8 1 , 8} \%$ & $18,2 \%$ \\
\hline Respiração & $\mathbf{7 2 , 7 \%}$ & $27,3 \%$ \\
\hline Medicação & $\mathbf{5 4 , 5 \%}$ & $45,5 \%$ \\
\hline Alimentação & $36,3 \%$ & $63,7 \%$ \\
\hline Disposiçäo & $\mathbf{7 2 , 7 \%}$ & $27,3 \%$ \\
\hline
\end{tabular}

Quadro 1: Variáveis do questionário sobre os componentes do estilo de vida após 10 semanas de intervenção

Obs: em negrito, os resultados de melhoras acima de 50\% na amostra avaliada de acordo com a percepção dos responsáveis

Por meio do Quadro 1, observa-se que as variáveis problema respiratório, sono, respiração, uso de medicação (interpretada como diminuição do uso de medicações) e disposição para a prática de atividades físicas apresentaram melhoras após o programa, de acordo com a percepção dos responsáveis pelas crianças e adolescentes. Vale destacar que as alterações referentes ao "problema respiratório", conforme consta no questionário são referentes aos sintomas apresentados pelas crianças durante o período de intervenção. Os achados do presente estudo são confirmados por estudo de Antes et al., (2008), no qual se verificou a melhora dos aspectos gerais da saúde de crianças asmáticas após intervenção com atividades semelhantes (natação e ginástica respiratória). As percepções de melhora, segundo Gualdi e Tumelero (2004), podem ser atribuídas à melhora das condições físicas, pois a prática de atividades físicas beneficia alguns aspectos, como aumento do apetite, melhora do sono, diminuição do número de drogas e promove sensação de bem-estar.

Um estudo que também investigou os efeitos de intervenções como cinesioterapia e hidroterapia, também utilizando questionários para 
avaliação de diversos aspectos da qualidade de vida de crianças asmáticas, foi o realizado por Surovenko et al. (2003 apud TAKETOMI; MARRA; SILVA, 2005). Neste estudo, os autores obtiveram como resultado uma melhoria na qualidade de vida das crianças, além de diminuição de crises e hospitalizações, o que corrobora os achados da presente pesquisa.

Rezende et al. (2006), em seu estudo, avaliaram a qualidade de vida de crianças asmáticas, por meio da percepção de seus pais, antes e depois de um programa de reabilitação pulmonar com atividades de alongamentos, fortalecimento de membros superiores, abdominais e exercícios aeróbicos num período de 24 sessões. Os questionários compreendiam os domínios da atividade física, sintomas e emoções e os autores não encontraram diferenças estatisticamente significativas entre pré e pós-teste. No entanto, as variações entre os itens envolvendo os questionários demonstraram tendência à melhora na qualidade de vida na visão dos pais sobre a asma das crianças relacionada ao programa de reabilitação. Os autores ainda ressaltam que os dados relacionando asma infantil, exercícios físicos e qualidade de vida demonstram que o condicionamento cardiorrespiratório tem papel fundamental no manejo clínico desta patologia, proporcionando um controle maior da doença. Nessa perspectiva, Pituch e Bruggeman (1982 apud MOISÉS, 2006) confirmam que prática de atividades físicas para pessoas asmáticas, além de reduzir as crises, também é efetiva na redução das faltas escolares, melhora a disposição e influencia na melhora das atitudes pessoais e sociais.

Quanto ao comportamento do fluxo expiratório, pode-se observar, no Gráfico 1, os valores do teste de expirometria relativos a 7 semanas de aulas. Vale lembrar que quanto maiores os valores apresentados pelos participantes, melhor a capacidade de expiração. O período de avaliação desta variável foi mais curto devido aos testes serem realizados no início das aulas e despenderem de tempo para realização por serem feitos de forma individual, o que atrasava o início das intervenções. Analisando o gráfico, nota-se que, após a primeira semana, houve uma queda do volume expiratório com valores abaixo de $280 \mathrm{~L} / \mathrm{min}$. Entre a segunda e a quarta semanas, ocorreram picos nos volumes expiratórios e, a partir da quinta semana, houve uma queda neste volume (abaixo de 290L/min), conforme os valores dos pontos de corte (mínimo e máximo). Apesar desta queda e das oscilações perceptíveis no gráfico, ao final do treinamento pode-se perceber re- 
sultados levemente superiores aos encontrados no início do programa, indicando uma tendência à melhora da ventilação pulmonar das crianças e adolescentes participantes após o programa.

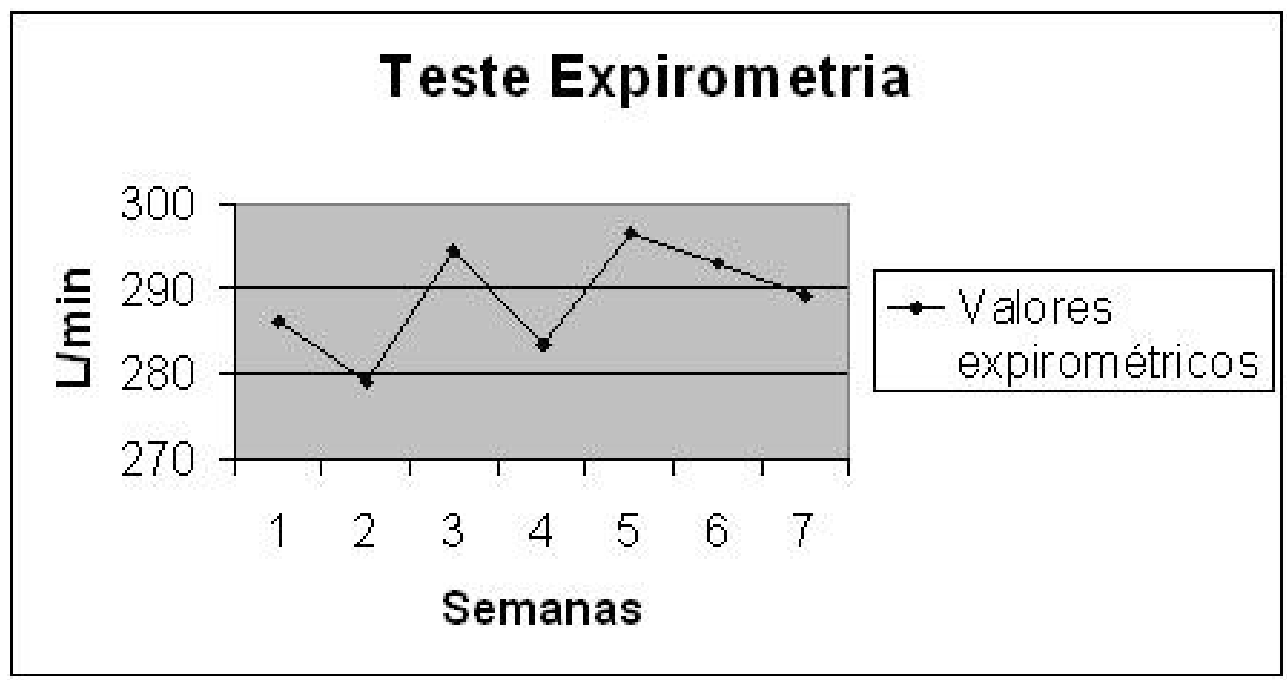

Gráfico 1: Valores referentes aos testes de expirometria no período de 7 semanas

Os resultados do presente estudo foram semelhantes aos obtidos em estudo de Moisés (2006), realizado com crianças e adolescentes na faixa etária entre 6 e 14 anos, de ambos os sexos submetidas a um programa de atividades desenvolvidas duas vezes na semana com duração de 45 minutos cada durante 5 meses. Constavam de atividades de ginástica respiratória (exercícios posturais, respiratórios e de relaxamento muscular) e natação. De acordo com a autora, as avaliações apresentaram resultados favoráveis para todas as faixas etárias, com redução no número de crises asmáticas durante o programa.

Outro estudo que também encontrou resultados positivos com a associação entre natação e ginástica respiratória foi o realizado por Silva, Santos e Miranda (1994). Utilizando programa com metodologia semelhante (natação e atividades fisioterápicas exercícios respiratórios, posturais e de relaxamento) os autores avaliaram os efeitos de atividades físico-recreativas para crianças asmáticas (8 aos 12 anos). Após a avaliação da capacidade aeróbica e mensuração do fluxo expiratório, os autores concluíram que as crianças, além de melhorarem a capacidade aeróbica, apresentaram uma atenuação qualitativa e quantitativa das crises asmáticas. Ainda, passaram a conviver melhor com as crises à medida que o domínio da técnica respiratória e das posturas corporais favoráveis amenizava o sofrimento provocado pelas crises. 
Nessa mesma linha, Moraes et al., (2007) avaliaram 93 crianças e adolescentes, de ambos os sexos, divididos em grupos GA $(n=69)$ e GC $(n=24)$ - grupo asmáticos e grupo controle (sem asma). Os grupo experimental (GA) participou de um programa de 7 meses de natação ( 2 vezes por semana) e foi avaliado o pico de fluxo expiratório em ambos os grupos. Os autores encontraram que as crianças do GA apresentaram os valores do Pico do Fluxo Expiratório (PFE), antes do programa, inferiores ao GC, no entanto, após o programa de natação, a variação percentual dos valores do PFE no GA foi maior do que no GC.

Outro estudo que também avaliou o fluxo expiratório de crianças asmáticas foi o realizado por Macedo Júnior et al. (2007), no qual foi avaliada se uma única sessão de natação promove alterações agudas pós-exercício do pico de fluxo expiratório em asmáticos. Participaram do estudo 8 meninos, com idades entre 5 e 14 anos, participando de uma sessão de natação com 45 minutos de duração com ênfase em atividades desobstrutivas da musculatura torácica, posturais e exercícios de respiração de natação, atividades estas semelhantes às desenvolvidas neste estudo. $\mathrm{O}$ fluxo expiratório foi medido em 3 momentos (antes do exercício, 10 minutos e 20 minutos após o exercício). Os autores concluíram que o efeito agudo do exercício de natação promove alterações favoráveis no pico de fluxo expiratório. Ao compararmos aos resultados do presente estudo, constatamos a importância de um período maior de atividades para manutenção e melhora da capacidade respiratória.

Gualdi e Tumelero (2004) realizaram um trabalho com exercícios aquáticos em indivíduos acometidos por asma, tendo, como finalidade, amenizar suas complicações. Fizeram parte do trabalho 4 crianças na faixa etária entre 5 e 9 anos, num programa de 3 meses com aulas de 45 minutos, 1 vez por semana. Os autores observaram melhora de $100 \%$ nos sintomas de asma, segundo as percepções dos responsáveis pelas crianças e, ainda, a intensidade das crises foi menor que quando não praticavam as atividades. Esses achados evidenciam que exercícios específicos para esta patologia, associados ao uso de medicamentos, são eficientes no controle da doença e melhora da condição física geral.

Em relação ao desempenho motor, os resultados individuais em pré e pós-testes podem ser observados pela análise do Gráfico 2. 


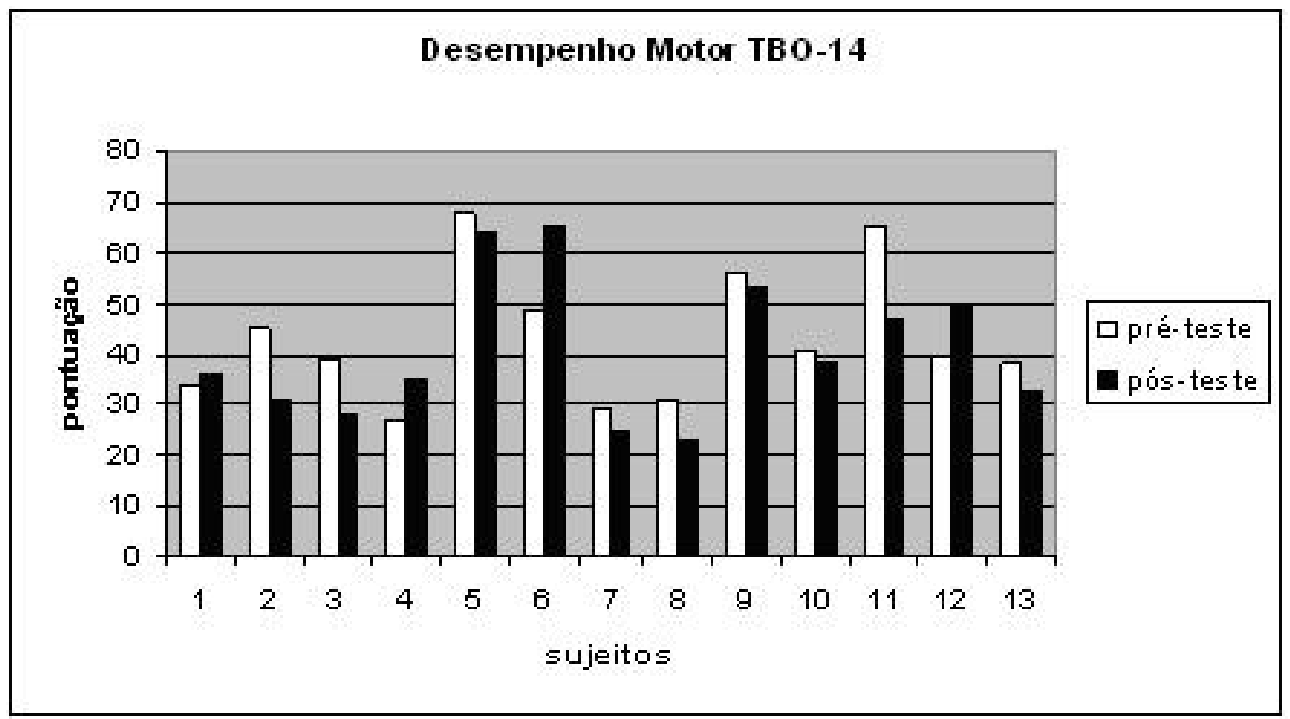

Gráfico 2: Desempenho motor TBO 14 - (Pontuação individual)

O Quadro 2 revela as médias do grupo para o desempenho motor em pré e pós-teste, apontando que os resultados em pós-testes foram inferiores aos obtidos em pré-teste.

\begin{tabular}{l|c|c}
\hline \multicolumn{1}{c|}{ Desempenho motor } & Média & DP \\
\hline Pré-teste & 43,23 & 13,06 \\
\hline Pós-teste & 40,62 & 13,98 \\
\hline
\end{tabular}

Quadro 2: Média e desvio-padrão em pré e pós-teste

Por meio da realização do teste $\mathrm{t}$ pareado, adotando nível de significância de $(p \leq 0,05)$, evidenciou-se que não houve diferença estatisticamente significativa $(\mathrm{p}=0,978)$ em relação às variáveis do desempenho motor antes e após o programa. Estes achados foram contrários às hipóteses do estudo, nas quais se acreditou que um programa de natação e ginástica respiratória proporcionaria melhorias no desempenho motor da população em questão. Um fator que pode ter influenciado o baixo desempenho no pós-teste foram as temperaturas baixas dos meses nos quais o estudo foi desenvolvido, já que o préteste foi realizado no início do mês de maio, no qual as temperaturas eram mais amenas.

Silva et al., (2005) avaliaram variáveis distintas às do presente estudo (condicionamento físico e força muscular) em crianças asmáticas na faixa etária entre 8 e 11 anos. O programa de exercícios físicos, com atividades semelhantes às realizadas no presente estudo, teve du- 
ração de 4 meses, com aulas de 90 minutos. Ao contrário dos achados do presente estudo, os autores encontraram diferenças estatisticamente significativas quando comparadas as avaliações iniciais e finais, evidenciando que um programa de treinamento físico específico, com sessões de 2 vezes semanais e duração de 90 minutos, propicia melhora no condicionamento físico e aumento da força muscular. Apesar do enfoque em diferentes variáveis, o programa do estudo anteriormente citado obteve resultados efetivos, o que pode ser atribuído ao prolongado período de duração das atividades que mantinham as crianças em movimento por mais tempo, refletindo na melhora de suas condições físicas.

Os resultados do presente estudo, apesar da modalidade distinta, podem ser comparados às constatações de Sá e Pereira (2003) que, em sua pesquisa, analisaram o equilíbrio e coordenação motora através do protocolo de Bruininski e Ozerestsky. Foram avaliadas crianças com idades entre 8 e 12 anos, do sexo masculino, iniciantes na prática de judô. Os autores também não encontraram diferença significativa entre os grupos experimentais (GE) e controle (GC) na capacidade equilíbrio após 36 semanas de treinamento físico específico para equilíbrio. Já em relação à capacidade coordenação motora, os resultados foram contrários aos do presente estudo, pois os autores encontraram diferenças significativas entre (GC) e (GE), evidenciando que um programa de treinamento físico específico simultâneo com as aulas de judô influencia positivamente para aumentar a performance de coordenação motora bilateral, tornando os movimentos mais harmoniosos

Outro estudo que também avaliou habilidades e padrões motores em asmáticos foi o realizado por Benites e Corazza (2003), utilizando o mesmo protocolo do presente estudo. O grupo foi composto por adolescentes portadores de asma brônquica $(n=31)$, com idades entre 11 e 14 anos, divididos em 5 grupos: (1) adolescentes com asma leve, participantes do projeto "Natação e ginástica respiratória para crianças e adolescentes asmáticos"; (2) adolescentes com asma moderada, participantes do projeto "Natação e ginástica respiratória para crianças e adolescentes asmáticos"; (3) adolescentes com asma leve, participantes de atividades físicas na escola; (4) adolescentes com asma moderada, participantes de atividades físicas na escola; (5) adolescentes com asma leve, não praticantes de programas de atividades físicas. Após avaliação de todas as crianças, os resultados revelaram que, independente dos estados clínicos (leve ou moderado) e da participação em 
atividades físicas em programas especiais ou na escola, a maioria dos sujeitos apresentou nível de habilidades motoras baixo e de padrão motor elementar. A partir da realização deste estudo, os autores atentam para a importância do conhecimento das restrições e possibilidades de desenvolvimento das crianças por parte dos profissionais que trabalham com essa população, a fim de que se elaborem atividades motoras estimuladoras que proporcionem melhor desenvolvimento e evolução das habilidades e padrões motores.

Barros et al., (2006) analisaram as variáveis neuromotoras (força de membros inferiores e agilidade) em 33 crianças de ambos os sexos, soropositivas, na faixa etária entre 7 e 12 anos. $O$ grupo avaliado foi composto de 11 crianças da periferia de São Paulo (não institucionalizadas) e 22 crianças moradoras em instituições próprias para pacientes soropositivos, todas elas participantes de aulas de Educação Física escolar, 2 a 3 vezes na semana. Os resultados encontrados nesta investigação mostraram que as crianças apresentaram comprometimento nas variáveis neuromotoras avaliadas quando comparadas a crianças soronegativas de uma escola pública de São Caetano. Foram encontradas diferenças estatisticamente significativas entre as institucionalizadas e não institucionalizadas, no entanto, quanto ao sexo, não houve diferença. De acordo com os autores, os comprometimentos nas variáveis analisadas no estudo podem ter resultado do comprometimento físico das crianças causado pelo próprio HIV e pelo tratamento medicamentoso, bem como por uma educação física com estimulação motora insuficiente para a melhora física necessária.

Ao analisarem-se as literaturas consultadas para realização da discussão deste estudo, concorda-se com os autores quando estes acreditam que uma maior preparação dos profissionais de Educação Física, na elaboração de atividades para crianças que apresentam certas fragilidades no seu desenvolvimento motor, é fundamental para que estas também sejam estimuladas durante as aulas e possibilitem, assim, oportunidade de melhora de suas capacidades e potencialidades. Esta premissa também é importante para os pais dessas crianças para que possibilitem, também no contexto doméstico, a exploração dos ambientes por meio de atividades e brincadeiras.

\section{Conclusão}

Os resultados deste estudo revelaram a eficiência do programa de 
ginástica respiratória e natação nos componentes do estilo de vida, a partir dos resultados positivos relatados pelas percepções dos pais ou responsáveis, evidenciando a importância do desenvolvimento de atividades físicas específicas para este grupo quanto à melhoria dos aspectos gerais da saúde.

Em relação ao comportamento do fluxo expiratório, os participantes apresentaram discreta melhora nos resultados após o programa e estão em conformidade com os achados de outros estudos que também evidenciaram os benefícios advindos da prática de atividades físicas para a melhora da função ventilatória em indivíduos asmáticos.

Atentando-se para os resultados da análise do desempenho motor, percebe-se que estes não foram condizentes com as expectativas do estudo, o que vai de encontro aos achados de outros estudos com crianças asmáticas com intervenções de exercícios respiratórios, posturais e de natação, que encontraram resultados efetivos de melhora.

As limitações encontradas no estudo são relativas ao período de desenvolvimento (10 semanas) que pode ter sido insuficiente para melhora nas demandas motoras. Ainda, não foram realizados exercícios motores específicos para cada variável avaliada (coordenação, agilidade, equilíbrio, etc.) através do protocolo.

Diante do exposto, percebe-se a importância da elaboração de programas motores com período maior (acima de 10 semanas) de duração e ainda específicos para o desenvolvimento de determinadas habilidades básicas, beneficiando também os vários aspectos da saúde geral dos indivíduos asmáticos.

Effect on regular practice for physical exercises regarding lifestyle and motor performance of asthmatic children and adolescents

\section{Abstract}

In this study, we aimed at verifying the effects of a 10-week respiratory exercise programme and swimming regarding lifestyle, motor performance and behaviour of expiratory flow in asthmatic children and adolescents. Thirteen subjects have participated in this study, and their mean age was $11.09 \pm 2.24$ years, including both genders - male and female. In order to assess the lifestyle, we used a questionnaire on how to use medication and conditions to do physical activities, improve sleep and suchlike. To verify the behaviour of expiratory flow, we used expirometer, and to assess the motor performance we used the Bruininks-Oseretsky Test of Motor Proficiency (BOTMP). The results showed improvements for the elements of lifestyle, as this study authors observed, and a slightly improvement for the expiratory flow after the programme. Regarding the motor performance, there was 
not statistically significant difference after the programme.

Keywords: Asthma - Children - Physical Activity - Lifestyle - Motor Performance

El efecto de la práctica regular de ejercicio físico en estilo de vida y rendimiento motor de niños y adolescentes asmáticos

\section{Resumen}

Este estudio ha verificado los efectos de un programa de gimnasia respiratoria y natación en el estilo de vida y desempeño motor de niños y adolescentes asmáticos. Participaron del estudio 13 individuos con edad media de 11,09 $\pm 2,24$ años, de ambos los sexos. Para la evaluación del estilo de vida se ha utilizado un cuestionario, para la verificación del comportamiento del flujo espiratorio se ha utilizado un espirómetro y para el análisis del desempeño motor la Batería de Testes de Bruininski y Ozerestsky. Los resultados han revelado mejorías en los elementos del estilo de vida, según la percepción de los responsables y una sutil mejora del flujo espiratorio tras el programa. Cuanto al desempeño motor no ha tenido diferencia estadísticamente significativa tras el programa.

Palabras clave: Asma - Niños - Actividad Física - Estilo de Vida - Desempeño Motor

\section{Referências}

ANTES, D. L. et al. Aspectos gerais da saúde em portadores de enfermidades respiratórias praticantes de um programa de atividades físicas regulares. Lecturas Educación Física y Deportes, Buenos Aires, v. 13, p. 1/123-2, 2008. Disponível em: <http://www.efdeportes.com> Acesso em: 20 dez. 2008.

BARROS, C. et al. Avaliação das variáveis de força muscular, agilidade e composição corporal em crianças vivendo com HIV/AIDS. Revista Brasileira Ciência e Movimento, Brasília, v. 14 n. 4, p. 47-54, 2006.

BENITES, A. C. F.; CORAZZA, S.T. Habilidades e padrões motores em adolescentes portadores de asma brônquica. Kinesis, n. 29, ago./dez./ 2003.

BETIO, J.; KREBS, R. J; KEULEN, G. V. Atividade física para portadores de asma. Cinergis, Santa Cruz do Sul, v. 8, n. 2, p. 7-12, jul./dez./ 2007.

BROCKMANN, P. V. et al. Actividad física y obesidad en ninõs con asma. Revista Chilena de Pediatria, Santiago, v. 78, n. 5, p. 482-488, 
2007.

CABRAL, A. L.; TEIXEIRA, L. R. (coord). Vencendo a asma: uma abordagem multidisciplinar. São Paulo: Bevilacqua, 1994.

CASSOL, V. E. et al. Broncoespasmo induzido pelo exercício em crianças e adolescentes com diagnóstico de asma. Revista Brasileira de Pneumologia, Rio de Janeiro, v. 30, n. 2, p. 102-108, 2004.

GALLAHUE, D. L. Cross-cultural considerations in the motor performance of young children. Indianápolis, 1994.

GALlAHUE, D. L.; OZMUN, J. C. Compreendendo o desenvolvimento motor: bebês, crianças, adolescentes e adultos. São Paulo: Phorte, 2005.

GUALDI, F. R.; TUMELERO, S. Asma e os benefícios da atividade física. Lecturas Educación Física y Deportes, Buenos Aires, año 10, n. 72, mayo/ 2004. Disponível em: <http://www.efdeportes.com> Acesso em: 23 ago. 2007.

MACEDO JÚNIOR, A. T. et al. Resposta aguda do pico de fluxo expiratório após uma sessão de exercícios de natação em crianças asmáticas. In: SIMPÓSIO NORDESTINO DE ATIVIDADE FÍSICA E SAÚDE, 6. Lauro de Freitas - BA. Anais, 2007.

MOISÉS, M. P. (coord). Atividades físicas e a criança asmática. Brasília: Ministério da Educação e do Desporto, Secretaria dos Desportos, 1993.

- Ginástica respiratória para asmáticos: efeito de redução do número e intensidade de crises asmáticas. Revista Mackenzie de Educação Física e Esporte, São Paulo, v. 5, n. esp., p. 76-81, 2006.

MORAES, G. M. L. et al. Comportamento do fluxo expiratório antes e após aula de natação em crianças portadoras de asma. Revista Sociedade Brasileira Clínica de Medicina, São Paulo, v. 5, n. 1, p. 7-13, jan./fev./ 2007.

PITANGA, F. J. G. Epidemiologia da atividade física, exercício físico e saúde. 2. ed. São Paulo: Phorte, 2004. 
REZENDE, I. M. O. et al. Efeitos da reabilitação pulmonar sobre a qualidade de vida: uma visão das crianças asmáticas e de seus pais. Acta Fisiatrica, São Paulo, v. 15, n. 3, p. 165-169, 2006.

SÁ, V. W.; PEREIRA, J. S. Influência de um programa de treinamento físico específico no equilíbrio e coordenação motora em crianças iniciantes no judô. Revista Brasileira Ciência e Movimento, Brasília, v. 11, n. 1, p. 45-52, 2003.

SILVA, C. S. et al. Avaliação de um programa de treinamento físico por quatro meses para crianças asmáticas. Revista Brasileira de Pneumologia, Rio de Janeiro, v. 31, n. 4, p. 270-285, 2005.

SILVA, F. M.; SANTOS, A. C.; MIRANDA, J. As atividades físicas na recuperação das crianças portadoras de deficiência respiratória. Revista do Centro de Ciências da Saúde, Santa Maria, v. 13, n. 4, p. 16-21, out-dez 1994.

SOCIEDADE BRASILEIRA DE PNEUMOLOGIA E TISIOLOGIA. Manual de pneumologia. Porto Alegre: SBPT, 2002.

TAKETOMI, E. A.; MARRA, S. M. G.; SILVA, G.R. Fisioterapia em asma: efeito da função pulmonar e em parâmetros imunológicos. Fitness \& Performance Journal, Rio de Janeiro, v. 4, n. 2, p. 97-100, mar./abr./ 2005.

WEISGERBER, M. C. et al. Benefits of swimming in asthma: effect of a session of swimming lessons on symptoms and PFTs with review of the literature. Journal of Asthma, v. 40, n. 5, p. 453-454, 2003.

Recebido em: 10/09/2009

Revisado em: 21/02/2010

Aprovado em: 13/04/2010

Endereço para correspondência

andressa_contreira@yahoo.com.br

Andressa Ribeiro Contreira

Avenida Roraima, 1000

Cidade Universitária, Prédio 51

Bairro Camobi

CEP: 97.105-900

Santa Maria, Rio Grande do Sul, Brasil 